\title{
A nomogram for bladder pain syndrome/interstitial cystitis based on netrin-1
}

\author{
Xiaojie Ang ${ }^{1} \cdot$ Yufeng Jiang ${ }^{1} \cdot$ Zongqiang Cai $^{1} \cdot$ Qi Zhou ${ }^{1} \cdot$ Miao Li $^{1} \cdot$ Bin Zhang ${ }^{1} \cdot$ Weiguo Chen $^{1} \odot \cdot$ Li-Hua Chen $^{3}$. \\ Xi Zhang ${ }^{2}$
}

Received: 8 September 2021 / Accepted: 4 December 2021 / Published online: 13 December 2021

(c) The Author(s) 2021

\begin{abstract}
Purpose This study aimed to combine plasma netrin-1 and clinical parameters to construct a diagnostic model for bladder pain syndrome/interstitial cystitis (BPS/IC).

Methods We analyzed the independent diagnostic value of netrin-1 and the correlation with clinical symptom scores of BPS/IC. Clinical parameters were selected using LASSO regression, and a multivariate logistic regression model based on netrin-1 was established, and then a nomogram of BPS/IC prevalence was constructed. The nomogram was evaluated using calibration curves, the C-index, and decision curve analysis (DCA). Finally, the model was validated using an internal validation method.

Results The area under the curve for the ability of netrin- 1 to independently predict BPS/IC diagnosis was $0.858(p<0.001)$, with a sensitivity of $85 \%$ and specificity of $82 \%$. The predicted nomogram included three variables: age, CD $3+/ C D 4+\mathrm{T}$ lymphocyte ratio, and netrin-1. The $\mathrm{C}$-index of this nomogram was 0.882 , and the predicted values were highly consistent with the actual results in the calibration curve. In addition, the internally validated C-index of 0.870 confirms the high reliability of the model. DCA results show that the net patient benefit of the netrin-1 combined with other clinical parameters was higher than that of the single netrin-1 model.

Conclusion Netrin-1 can be used as a diagnostic marker for BPS/IC and is associated with pain. The nomogram constructed by combining netrin-1 and clinical parameters was able to predict BPS/IC with great accuracy. In addition, Netrin-1 may also serve as a novel therapeutic target for BPS/IC.
\end{abstract}

Keywords Bladder pain syndrome $\cdot$ Interstitial cystitis $\cdot$ Netirn- $1 \cdot$ Logistic regression model $\cdot$ Nomogram

Xiaojie Ang, Yufeng Jiang, and Zongqiang Cai are co-first authors

Xiaojie Ang, Yufeng Jiang, and Zongqiang Cai are co-first authors
of the article.

\footnotetext{
Weiguo Chen

chenweiguo1971@suda.edu.cn

$\bowtie$ Li-Hua Chen

lhchen@ntu.edu.cn

faceza@163.com

1 Department of Urology, The First Affiliated Hospital of Soochow University, 188 Shizi Street, Suzhou 215006, Jiangsu Province, China

2 Department of Urology, Kunshan Hospital of Traditional Chinese Medicine, No 189 Chao Yang Road, Kunshan, Jiangsu, China

3 Department of Nutrition and Food Hygiene, School of Public Health, Nantong University, Jiangsu, China
}

$\triangle$ Xi Zhang

\section{Introduction}

Bladder pain syndrome/interstitial cystitis (BPS/IC) is a chronic condition of recurrent lower abdominal discomfort or pelvic pain associated with bladder filling in the absence of a urinary tract infection [1]. BPS/IC is associated with age, gender, and race, and the incidence of BPS/IC is $2-5$ times higher in women than in men [2]. Currently, diagnosis of BPS/IC is based on clinical symptoms and staging based on cystoscopy to identify Hunner's ulcers in the bladder mucosa; an obvious drawback of such a test is its invasiveness [3]. In recent decades, potential biomarkers of BPS/IC have been sought using proteomics [4]. Nevertheless, no ideal single or combination of biomarkers has been identified.

Netrin-1 is a soluble protein secreted by basal plate cells; it was the first neural-specific factor identified in the netrin 
family $[5,6]$. Netrin- 1 is expressed in the nervous system and organs, such as the lung, pancreas, mammary gland, and intestinal epithelium [7]. Recent studies have revealed that netrin-1, a novel neuro-immune inflammatory factor, regulates the migration of inflammatory cells such as macrophages and the secretion of inflammatory factors [8]. In addition, netrin- 1 has been associated with inflammatory pain in osteoarthritis and chronic pelvic pain associated with endometriosis $[9,10]$. BPS/IC, a sterile inflammatory disease, has chronic pelvic pain as one of its main symptoms, and it is unclear whether netrin- 1 is involved in the pathogenesis of BPS/IC.

In the present study, we aimed to investigate the diagnostic value of netrin-1 for BPS/IC and its clinical relevance. First, we examined netrin-1 expression in BPS/IC and analyzed the correlation between its expression and pain scores. Second, we constructed a novel nomogram prediction model based on netrin- 1 and verified the reliability of the model.

\section{Methods}

\section{Study design and participants}

The Ethics Committee of the First Affiliated Hospital of Soochow University approved the study (approval number: 2020027), and all subjects provided written informed consent. The diagnosis of BPS/IC was based on the criteria established by the US National Institute of Diabetes and Digestive and Kidney Diseases [11]. Sixty women with nonulcerated BPS/IC diagnosed by cystoscopy at the First Hospital of Soochow University between 03/2020 and 02/2021 were included in the BPS/IC group, and 60 healthy women undergoing check-ups at our hospital during the same period were selected as the control group. Healthy patients were defined as those with no history of disease other than diabetes or hypertension.

The inclusion criteria were as follows: (1) age between 18 and 70 years; (2) urination $>8$ times during wakefulness, $\geq 2$ episodes of nocturia per night, and $\geq 9$ months duration of the condition; (3) bladder filling accompanied by discomfort in the pelvic area, perineum, lumbar region, or urethral vulva, with significant relief of pain or discomfort after urination; (4) bladder volume $<350 \mathrm{~mL}$; (5) Hunner's ulcer or glomerular-like bleeding spot visible on cystoscopy, meeting the diagnostic criteria.

The exclusion criteria were as follows: (1) symptoms relieved with the application of antibiotics or antispasmodics; (2) association with diseases, such as urinary stones, urinary tract infections, or urinary tract tumors; (3) nonprimary patients who received appropriate medication and had improved symptoms; (4) pregnant women and patients with allergies.

\section{Data collection}

All subjects underwent testing for complete blood counts, C-reactive protein (CRP), and CD3 + CD4 + T lymphocyte ratio. $2 \mathrm{ml}$ of blood was left to stand for $30 \mathrm{~min}$ and then centrifuged ( $3000 \mathrm{r} / \mathrm{min}$ for $10 \mathrm{~min}$ ) and circulating levels of netrin-1 were evaluated with commercial ELISA kits (CSB-E11899h, Cusabio Biotech, Wuhan, China). The sensitivity of the ELISA kit was $7.81 \mathrm{pg} / \mathrm{ml}$, with intra- and inter-batch coefficients of variation of less than $8 \%$ and $10 \%$, respectively.

Participants' information including age, height, weight, diabetes or hypertension was collected. Questionnaires about O'Leary-Sant scale problem index (ICPI), the interstitial cystitis symptom index (ICSI), and a visual analog scale (VAS) were administered to patients in the BPS/IC group.

\section{Logistic regression modeling}

The "glmnet" package in R 4.0.0 software was used to select clinical characteristics using LASSO regression for 120 subjects with regard to age, BMI, hypertension, diabetes, menopause, CRP level, neutrophil-to-lymphocyte ratio (NLR), $\mathrm{CD} 3+\mathrm{CD} 4+\mathrm{T}$ lymphocyte ratio, and netrin-1 level. LASSO regression was used to select the most important features to avoid overfitting or overcomplicating the model $[12,13]$. The independent variables obtained from the LASSO regression screening were incorporate into the multivariate logistic regression model [14].

\section{Construction and evaluation of nomograms}

The "rms" package in the R 4.0.0 software was used to create nomograms based on the contribution of each variable in the logistic regression model to the outcome variables [15]. After plotting the nomogram, the consistency C-index of the model was calculated, and the nomogram was evaluated using the graphical calibration method. The receiver operating characteristic (ROC) curve for a single netrin-1 diagnosis and decision curve analysis (DCA) for netrin-1 combined with other clinical parameters were plotted using the "pROC" and "rmda" packages in R 4.0.0 software, respectively, to compare the advantages and disadvantages of the models [16].

\section{Statistical methods}

Statistical analysis was performed using the R software (Version 4.0.0; https://www.R-project.org). The mean \pm standard deviation $(x \pm s)$ was used for those with normal distribution, while the median and interquartile range were used for 
skewed distribution. The ROC of each parameter was plotted using the R software package "pROC" to determine the diagnostic value, and the correlations between netrin-1 and BPS/IC were analyzed using Spearman's rank correlations. Clinical characteristics were then selected using LASSO regression, logistic regression models were constructed and Nomograms were plotted. The accuracy of the model was assessed using C-index, calibration curves, ROC and DCA. Finally, the accuracy of the prediction model was verified by calculating the $\mathrm{C}$-index using an internal validation method (1000 Bootstrap self-sampling) [17]. Differences were considered statistically significant at $P<0.05$.

\section{Results}

\section{Characteristics of the study participants}

The mean ages of the BPS/IC and control groups were $50.30 \pm 10.34$ years (26-68 years) and $51.53 \pm 11.11$ years (25-69 years). Cystoscopic Hunner's ulcers were not seen in any patient in the BPS/IC group. There were no significant differences between groups with regard to age, BMI, CRP, or NLR. The mean values of CD3 + CD4 + T lymphocyte ratio were $41.48 \pm 6.18 \%$ and $37.38 \pm 2.71 \%$ in the two groups, respectively $(P<0.001$, Table 1$)$.

The plasma netrin-1 levels in the BPS/IC and control groups were $847.30 \pm 654.55 \mathrm{pg} / \mathrm{ml}$ and $373.00 \pm 167.51 \mathrm{pg} /$ $\mathrm{ml}$, respectively $(P<0.001$, Fig. $1 \mathrm{~A})$. The area under the ROC curve (AUC) for the ability of netrin-1 level to independently predict a diagnosis of BPS/IC was 0.858 (95\% CI: 0.788 to $0.929, P<0.001$ ), with better diagnostic efficacy than other clinical parameters including age, BMI, CRP, and NLR (Fig. 1B). The optimal cut-off value was $443.46 \mathrm{pg} /$ $\mathrm{ml}$ (the sensitivity was $85 \%$, and the specificity was $82 \%$ ).

\section{Correlation between netrin-1 and clinical symptom scores}

The correlation coefficients between plasma netrin-1 levels and patient ICSI, ICPI, VAS score, and maximum bladder capacity in BPS/IC patients were $0.63,0.68,0.59$, and -0.57 , respectively. There were moderately positive correlations between plasma netrin-1 levels and patient ICSI, ICPI, and VAS in BPS/IC patients and a moderate negative correlation with maximum bladder capacity (Fig. 2A-D).

\section{Logistic regression model}

Screening the clinical parameters using LASSO regression revealed that three independent variables were included in the model: age (older than 55 years), $\mathrm{CD} 3+\mathrm{CD} 4+\mathrm{T}$ lymphocyte ratio, and netrin-1 levels (Fig. 3). A multivariate logistic regression model was developed based on these variables (Table 2 ). $\mathrm{CD} 3+\mathrm{CD} 4+\mathrm{T}$ lymphocyte ratio and netrin-1 were independent risk factors $(P<0.001)$. For three variable axes (age, CD3 + CD4 + T lymphocyte ratio, and netrin-1 level), the corresponding points on the axes correspond to different scores. The scores of each predictive variable are summed to obtain a total score. Different total scores correspond to different probabilities of BPS/IC incidence (Fig. 4).

\section{Construction and evaluation of nomograms}

The calibration curve showed that the predicted incidence of BPS/IC was close to the actual results (the C-index of the model was 0.882 , which is a high predictive value) (Fig. 5). The C-index of the model was 0.870 after validation of the diagnostic prediction model using internal validation, and the model has excellent predictive performance.
Table 1 Comparison of various clinical characteristics between the two groups

\begin{tabular}{|c|c|c|c|c|}
\hline \multirow[t]{2}{*}{ Characteristics } & \multicolumn{2}{|l|}{ Group } & \multicolumn{2}{|c|}{ Significance test } \\
\hline & $\mathrm{BPS} / \mathrm{IC}(n=60)$ & Control $(n=60)$ & $t / \chi^{2}$ & $P$-value \\
\hline Age (years) & $50.30 \pm 10.34$ & $51.53 \pm 11.11$ & -0.630 & 0.530 \\
\hline $\mathrm{BMI}\left(\mathrm{kg} \cdot \mathrm{m}^{-2}\right)$ & $22.86 \pm 3.11$ & $22.72 \pm 2.16$ & 0.280 & 0.780 \\
\hline $\mathrm{CD} 3+\mathrm{CD} 4+(\%)$ & $41.48 \pm 6.18$ & $37.38 \pm 2.71$ & 4.704 & $<0.001$ \\
\hline $\mathrm{CRP}(\mathrm{mg} / \mathrm{L})$ & $0.99 \pm 1.03$ & $0.97 \pm 0.61$ & 0.121 & 0.904 \\
\hline NLR & $2.25 \pm 1.03$ & $2.21 \pm 0.73$ & 0.248 & 0.805 \\
\hline \multicolumn{5}{|c|}{ Comorbidities, number of case (\%) } \\
\hline Hypertension & $17(28.3)$ & $21(35.0)$ & 0.616 & $0.432^{\mathrm{a}}$ \\
\hline Diabetes & $17(28.3)$ & $16(26.7)$ & 0.042 & $0.838^{\mathrm{a}}$ \\
\hline Pausimenia & $32(53.3)$ & $32(53.3)$ & 0.000 & $1.000^{\mathrm{a}}$ \\
\hline
\end{tabular}

Analysis by paired $t$ tests

$B M I$ body mass index; $C R P$ C-reactive protein; $N L R$ neutrophil-to-lymphocyte ratio

${ }^{\mathrm{a}}$ Cardinality test (Monte Carlo significance) 


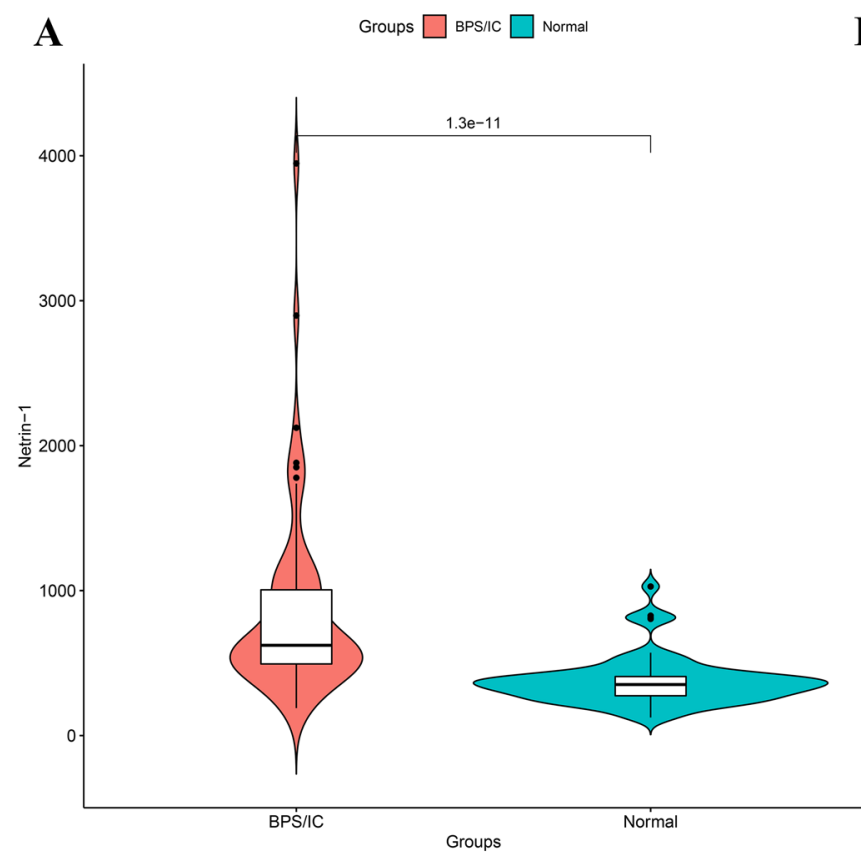

Fig. 1 A Violin plot of plasma netrin-1 levels in the BPS/IC and control groups. Plasma netrin-1 levels in the BPS/IC group were significantly higher than those in the control group, $P<0.001$. B Comparison of the diagnostic efficacy of various clinical parameters for BPS/

The AUC of ROC for the ability of netrin- 1 level to independently predict a diagnosis of BPS/IC was $0.858(95 \%$ CI: $0.788-0.929, P<0.001$ ), and the AUC of ROC for the nomogram model of netrin- 1 combined with other clinical parameters was 0.884 (95\% CI: 0.821-0.946, $P<0.001$ ), with a sensitivity of $80 \%$ and specificity of $90 \%$. The diagnostic efficacy of the nomogram model of netrin- 1 combined with other clinical parameters was slightly better than that of netrin-1 alone (Fig. 6).

Decision curve analysis (DCA) showed that the threshold probability range for patients was $10-85 \%$ for netrin-1's ability to independently diagnose BPS/IC and 3-96\% for the diagnostic model with netrin-1 combined with other clinical parameters. The net benefit to patients when using the diagnostic prediction model with netrin-1 combined with other clinical parameters was significantly better than that of netrin-1 alone (Fig. 7).

\section{Discussion}

In this study, we made two critical clinical findings. First, netrin- 1 can be used as a biomarker for BPS/IC. Second, the diagnostic model of netrin-1 combined with clinical parameters has high predictive value. To our knowledge, this study is the first report to describe a diagnostic model for BPS/IC using netrin-1.

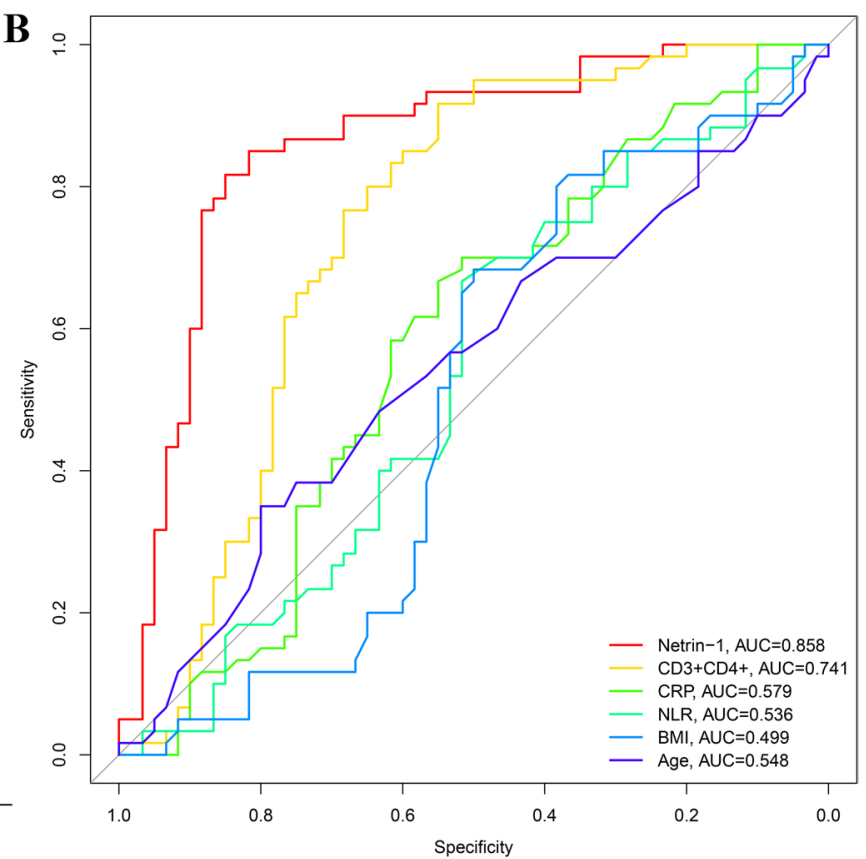

IC, in which netrin-1 and CD3 + CD4 + T lymphocyte ratio had good diagnostic efficacy, while age, BMI, CRP, and NLR had relatively poor diagnostic value

$\mathrm{BPS} / \mathrm{IC}$ is a chronic functional disorder characterized by hypersensitivity reactions in the lower urinary tract [18]. Approximately 8 million women worldwide may currently suffer from BPS/IC, and perhaps many more remain undiagnosed [19]. Therefore, the early, rapid, and accurate diagnosis of BPS/IC needs to be addressed. Many markers in BPS/ IC serum and urine have been described in previous studies; however, all have shortcomings. The sensitivity of GP51 in the urine of BPS/IC patients is too low. Anti-proliferative factor (APF) is the most sensitive and specific diagnostic marker for BPS/IC with a sensitivity of $94 \%$ and specificity of $95 \%$; however, it is not yet widely used in clinical practice due to the complexity of the APF assay technique. Nerve growth factor in urine is simple to measure; however, it is not sufficiently specific, and levels may be increased in the presence of urinary stones, urinary tract infection, or bladder outlet obstruction [20-22].

Serum and plasma specimens are less susceptible to contamination than urine specimens and better reflect the systemic nature of the disease. It has been shown that serum levels of serum pro-inflammatory cytokines (interleukins 1-beta and 6 and tumor necrosis factor-alpha) and chemokines (interleukin 8) are significantly higher in patients with BPS/ IC than in controls; However, these are less sensitive and specific [23, 24]. There is a growing consensus that BPS/IC is associated with neuro-immune inflammation, that pain associated with BPS/IC is considered a form of neuropathic 

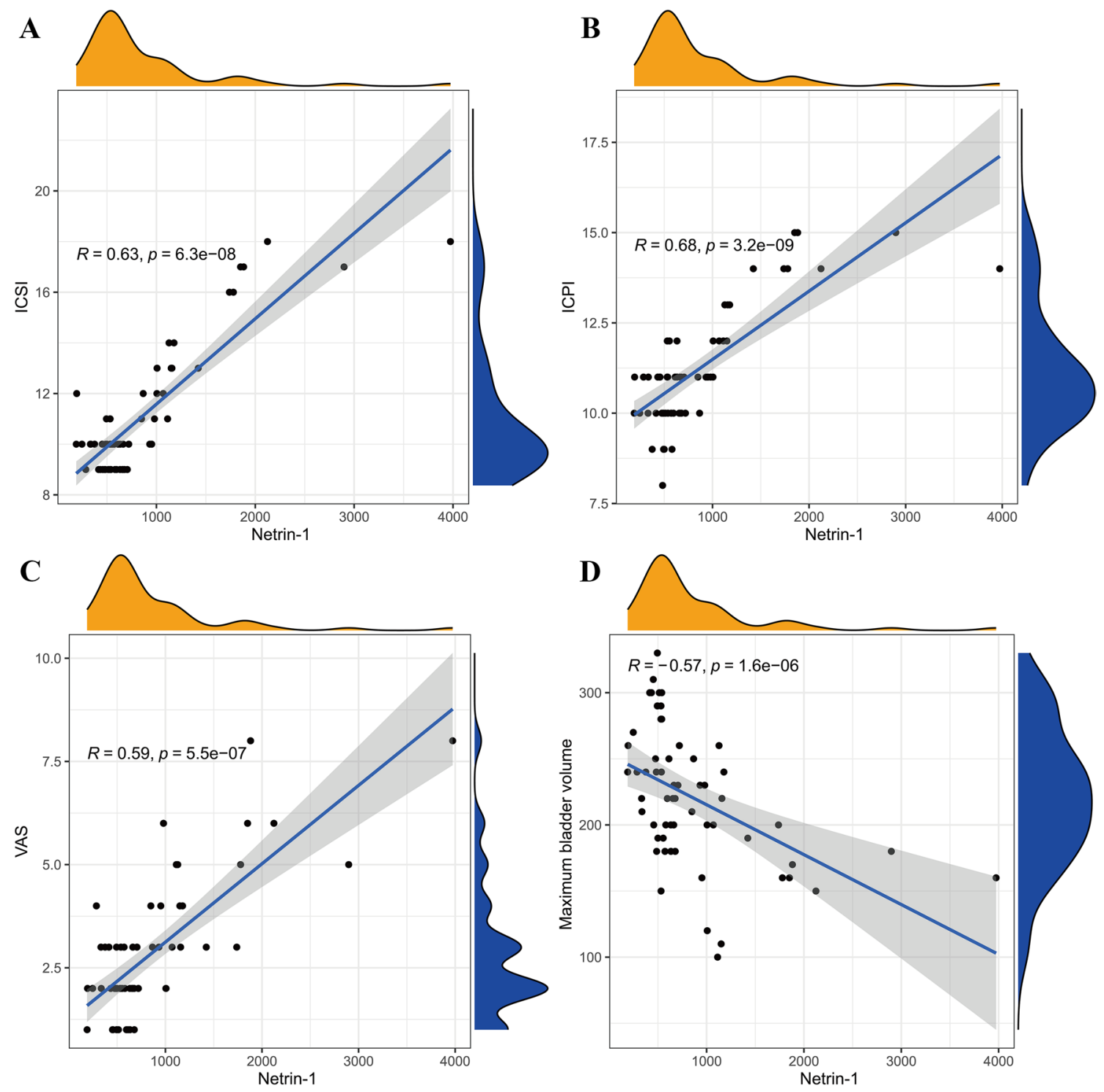

Fig. 2 Correlation analysis between netrin-1 and BPS/IC clinical symptom scores. A Netrin-1 correlated with ICSI with a coefficient of 0.63 , a moderately positive correlation; B Netrin- 1 correlated with ICPI with a coefficient of 0.68 , a moderately positive correlation; $\mathbf{C}$

Netrin-1 correlated with VAS with a coefficient of 0.59 , a moderate positive correlation; D Netrin-1 correlated with maximum bladder capacity with a coefficient of -0.57 , a moderately negative correlation

pain. Although growing evidence showed that the novel neuro-immune inflammatory factor netrin-1 regulated pain, there are no relevant studies of netrin-1 in BPS/IC [25, 26]. The potential biological relevant role of netrin-1 with BPS/ IC and easily measurable by commercial available kit build our rationale to study netrin- 1 levels in BPS/IC serum. Our results showed that the AUC of the ROC for was 0.858 (sensitivity of $85 \%$, specificity of $82 \%$ ) for netrin-1 to diagnose of BPS/IC. Netrin-1 was also correlated significantly with ICSI, ICPI scores, VAS scores, and maximum bladder capacity. As ICSI and ICPI scores increased and symptoms worsened, netrin-1 levels increased accordingly. Therefore, netrin-1 could be a potential molecular diagnostic marker

for BPS/IC, and elevated netrin-1 in plasma of women with BPS/IC is positively correlated with pain scores. This may provide a novel target and therapeutic idea for the management of BPS/IC, and the related mechanism deserves further investigation.

Logistic regression models are potent tools often used in clinical outcome studies. When applied uncritically, these models may generate results that do not fit well with the available data set or that do not accurately predict the outcome of a study. Therefore, it is necessary to perform LASSO regression to screen variables before building a logistic regression model to avoid poor fitting or overfitting $[27,28]$. In the present study, we constructed a rapid 

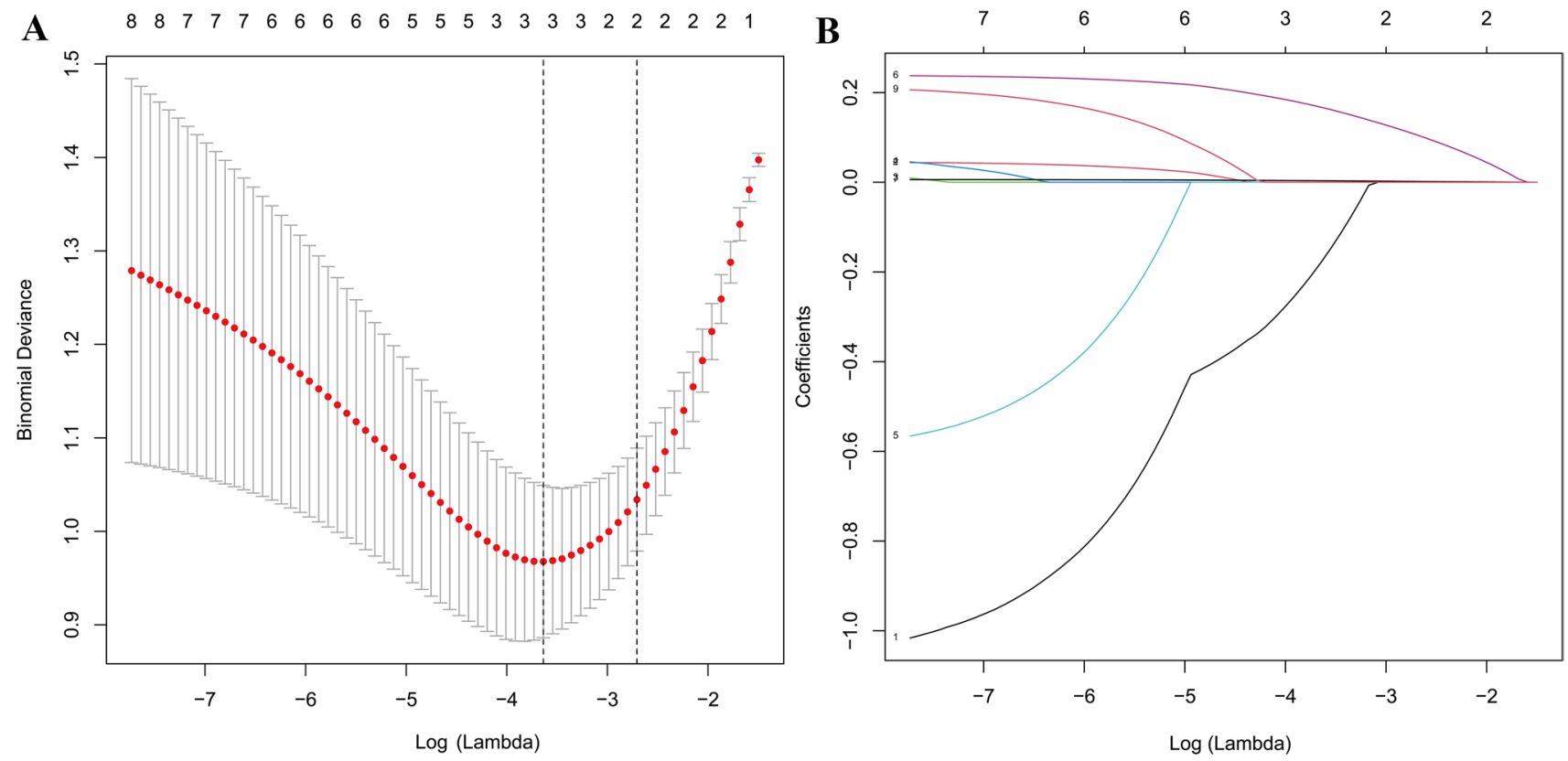

Fig. 3 Screening predictors for LASSO regression analysis. A The vertical line plotted at the optimal parameter lambda $=0.0182$ with three selected variables. B The plot of each clinical characteristic coefficient versus $\log ($ lambda) by adjusting the parameter lambda value

Table 2 BPS/IC multivariate logistic regression analysis

\begin{tabular}{lrrr}
\hline Intercept and variable & \multicolumn{2}{l}{ Prediction model } & \\
\cline { 2 - 4 } & \multicolumn{1}{c}{$\beta$} & Odds ratio (95\% CI) & $P$-value \\
\hline Intercept & -11.9891 & $0.000(0.000-0.001)$ & $<0.001$ \\
Age & -0.5749 & $0.563(0.204-1.485)$ & 0.252 \\
CD3+CD4+(\%) & 0.2399 & $1.271(1.114-1.494)$ & 0.001 \\
Netrin-1 & 0.0058 & $1.006(1.003-1.009)$ & $<0.001$ \\
\hline
\end{tabular}

Note: $\beta$ is the regression coefficient

and straightforward clinical diagnostic prediction model with an AUC of 0.884 under ROC, a sensitivity of $80 \%$, a specificity of $90 \%$, and a DCA with a threshold probability interval 3-96\%. The internal validation results suggest that the model has a discriminant $\mathrm{C}$-index of 0.870 and excellent model predictive performance. The AUC of the ROC improved slightly when comparing netrin- 1 alone with netrin-1 combined with other clinical parameters for prediction of BPS/IC. However, DCA showed a more significant difference, with a higher net benefit to patients from the diagnostic prediction model of netrin-1 combined with other clinical indicators. DCA is a simple method for evaluating clinical predictive models, diagnostic tests and molecular biomarkers [29]. In contrast to traditional performance measures, such as sensitivity, specificity and AUC, which failed to take into account the clinical utility of a particular model, DCA plots net benefit at a range of clinically reasonable risk thresholds, which can help to assess the utility of models for decision-making [30].

\section{Conclusion}

Overall, netrin-1 is a relatively accurate and valuable marker, and the nomogram model constructed based on netrin-1 can predict BPS/IC with great accuracy to assist physicians in making better clinical decisions. And the greater significance of this study is that it provides a new direction for research into the etiology and pathophysiology of BPS/IC. However, large data samples and multicentre studies are needed to validate our findings. 
Fig. 4 Nomogram of the BPS/ IC diagnostic prediction model for netrin-1 combined with clinical parameters. Note:

For three variable axes (age, $\mathrm{CD} 3+\mathrm{CD} 4+\mathrm{T}$ lymphocyte ratio, and netrin-1 level), the corresponding points on the axes correspond to different scores. The scores of each predictive variable are summed to obtain a total score. Different total scores correspond to different probabilities of BPS/IC prevalence
Points

$\begin{array}{lllllllllll}0 & 10 & 20 & 30 & 40 & 50 & 60 & 70 & 80 & 90 & 100\end{array}$

Age

$\underset{>=55}{<55}$

$\mathrm{CD} 3+\mathrm{CD} 4+$

$\begin{array}{llllllll}25 & 30 & 35 & 40 & 45 & 50 & 55 & 60\end{array}$
Netrin-1

Total Points

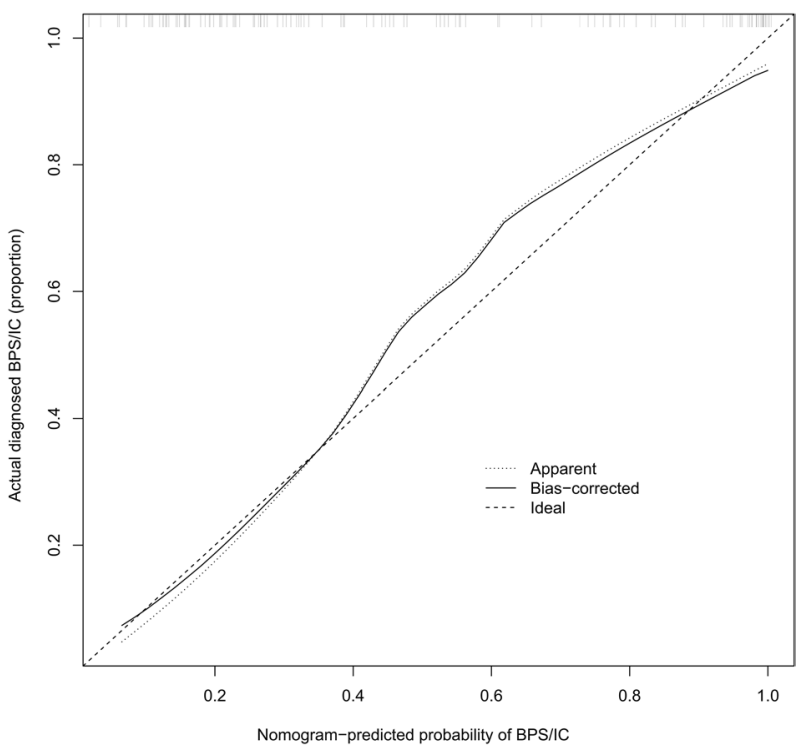

Fig. 5 Calibration curve of the BPS/IC nomogram model for netrin-1 combined with other clinical parameters. Note: The $x$-axis is the predicted BPS/IC occurrence probability of the nomotube model, and the $y$-axis is the actual BPS/IC occurrence rate. The diagonal dashed line represents the ideal model prediction performance; the solid line represents the prediction performance of the nomotube model in this study. The closer it is to the diagonal dashed line, the better the prediction performance
$\begin{array}{llll}0.1 & 0.30 .50 .7 & 0.9\end{array}$
0.9999

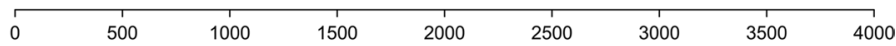

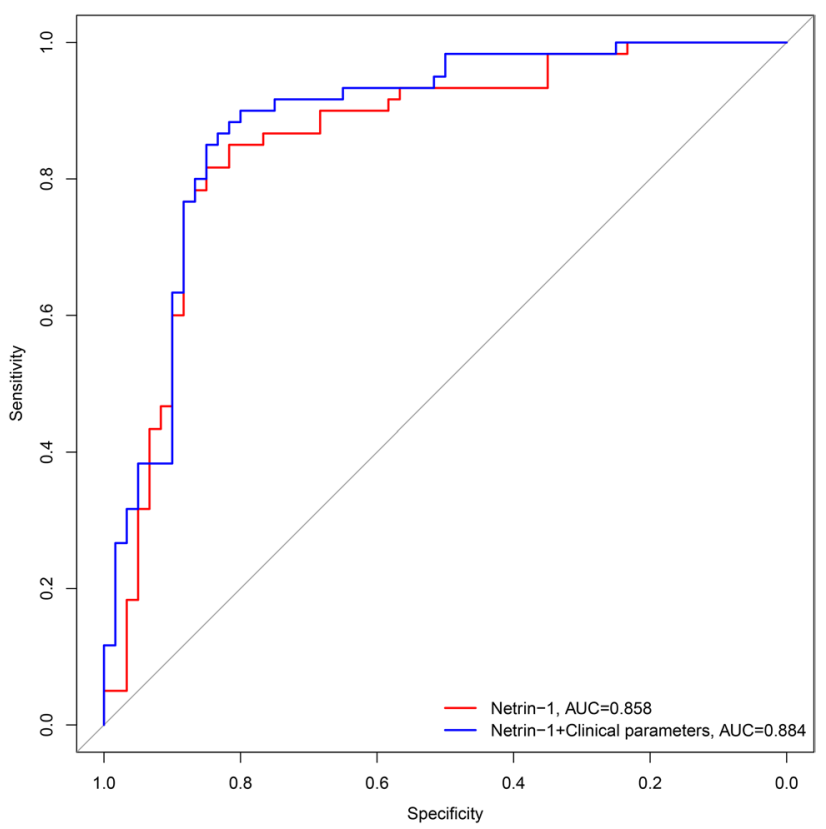

Fig. 6 ROC for the diagnosis of BPS/IC with the nomogram model for single netrin-1 and netrin-1 combined with other clinical parameters. Note: The two curved diagonal lines in the figure represent two different clinical diagnostic models (see legend label). Netrin-1 alone diagnosed BPS/IC with an AUC at ROC of 0.858 (95\% CI: 0.788 $0.929, P<0.001)$ and the nomogram model with netrin- 1 combined with other clinical parameters had an AUC of the ROC of $0.884(95 \%$ CI: $0.821-0.946, P<0.001) .95 \%$ CI: $0.821-0.946, P<0.001)$. The diagnostic efficacy of the nomogram model with netrin-1 combined with other clinical parameters was slightly better than that of netrin-1 alone 


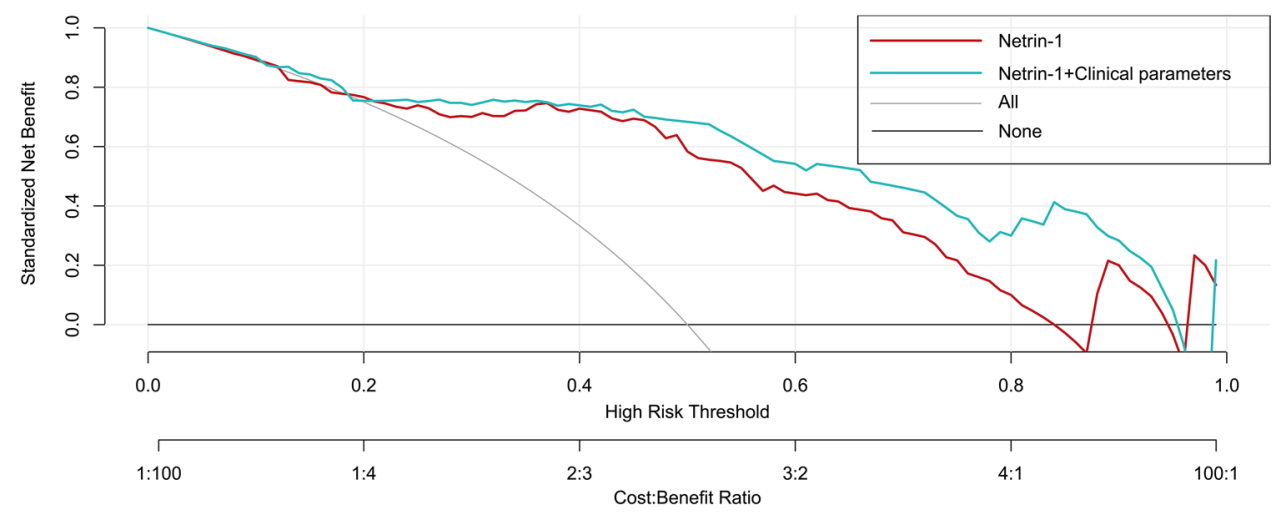

Fig. 7 DCA for the diagnosis of BPS/IC with a single netrin-1 and netrin-1 combined with other clinical parameters of the nomogram model. Note: The $x$-axis is the threshold probability, the $y$-axis is the net benefit normalized by prevalence, and the two curved sloping lines in the figure represent two different clinical diagnostic models

Author contributions (I) Conception and design: WC, L-HC; (II) Administrative support: XZ, WC; (III) Provision of study materials: ML; (IV) Collection and assembly of data: XA, YJ, ZC; (V) Data analysis and interpretation: XA, QZ; (VI) Manuscript writing: All authors; (VII) Final approval of manuscript: All authors.

Funding This study was supported by National Natural Science Foundation of China (grant: 81803307).

Data availability The datasets generated and analysed during the current study are not publicly available due to protect patient privacy.

Code availability Not applicable.

\section{Declarations}

Conflict of interest The authors have no conflicts of interest to declare.

Ethical approval The Ethics Committee of the First Affiliated Hospital of Soochow University approved the study (NO. 2020027) and informed consent was taken from all individual participants. All participants gave informed consent before participation.

Consent to participate All participants gave consent.

Consent for publication Manuscript is approved by all authors for publication.

Open Access This article is licensed under a Creative Commons Attribution 4.0 International License, which permits use, sharing, adaptation, distribution and reproduction in any medium or format, as long as you give appropriate credit to the original author(s) and the source, provide a link to the Creative Commons licence, and indicate if changes were made. The images or other third party material in this article are included in the article's Creative Commons licence, unless indicated otherwise in a credit line to the material. If material is not included in the article's Creative Commons licence and your intended use is not (see legend). The net benefit for patients in the diagnostic prediction model with netrin- 1 combined with other clinical parameters is significantly higher than the net benefit for patients diagnosed with netrin- 1 alone

permitted by statutory regulation or exceeds the permitted use, you will need to obtain permission directly from the copyright holder. To view a copy of this licence, visit http://creativecommons.org/licenses/by/4.0/.

\section{References}

1. Akiyama Y, Hanno P (2019) Phenotyping of interstitial cystitis/ bladder pain syndrome. Int J Urol 26(Suppl 1):17-19

2. Akiyama Y, Homma Y, Maeda D (2019) Pathology and terminology of interstitial cystitis/bladder pain syndrome: a review. Histol Histopathol 34(1):25-32

3. Patnaik SS, Laganà AS, Vitale SG, Butticè $S$, Noventa M, Gizzo S et al (2017) Etiology, pathophysiology and biomarkers of interstitial cystitis/painful bladder syndrome. Arch Gynecol Obstet 295(6):1341-1359

4. Argade S, Chermansky C, Tyagi P (2016) Biomarkers for interstitial cystitis/painful bladder syndrome. Womens Health (Lond) 12(1):87-90

5. Varadarajan SG, Kong JH, Phan KD, Kao TJ, Panaitof SC, Cardin J et al (2017) Netrin1 produced by neural progenitors, not floor plate cells, is required for axon guidance in the spinal cord. Neuron 94(4):790-9.e3

6. Kennedy TE, Serafini T, de la Torre JR, Tessier-Lavigne M (1994) Netrins are diffusible chemotropic factors for commissural axons in the embryonic spinal cord. Cell 78(3):425-435

7. Jiang YH, Jhang JF, Hsu YH, Ho HC, Wu YH, Kuo HC (2021) Urine biomarkers in ESSIC type 2 interstitial cystitis/bladder pain syndrome and overactive bladder with developing a novel diagnostic algorithm. Sci Rep 11(1):914

8. Toque HA, Fernandez-Flores A, Mohamed R, Caldwell RB, Ramesh G, Caldwell RW (2017) Netrin-1 is a novel regulator of vascular endothelial function in diabetes. PLoS ONE 12(10):e0186734

9. Ding S, Guo X, Zhu L, Wang J, Li T, Yu Q et al (2021) Macrophage-derived netrin-1 contributes to endometriosis-associated pain. Ann Transl Med 9(1):29

10. Zhu S, Zhu J, Zhen G, Hu Y, An S, Li Y et al (2019) Subchondral bone osteoclasts induce sensory innervation and osteoarthritis pain. J Clin Invest 129(3):1076-1093 
11. Malde S, Palmisani S, Al-Kaisy A, Sahai A (2018) Guideline of guidelines: bladder pain syndrome. BJU Int 122(5):729-743

12. Alhamzawi R, Ali HTM (2018) The Bayesian adaptive lasso regression. Math Biosci 303:75-82

13. Alhamzawi R, Alhamzawi A, Mohammad Ali HT (2019) New Gibbs sampling methods for bayesian regularized quantile regression. Comput Biol Med 110:52-65

14. Meurer WJ, Tolles J (2017) Logistic regression diagnostics: understanding how well a model predicts outcomes. JAMA 317(10):1068-1069

15. Park SY (2018) Nomogram: an analogue tool to deliver digital knowledge. J Thorac Cardiovasc Surg 155(4):1793

16. Van Calster B, Wynants L, Verbeek JFM, Verbakel JY, Christodoulou E, Vickers AJ et al (2018) Reporting and interpreting decision curve analysis: a guide for investigators. Eur Urol 74(6): 796-804

17. Wang H, Zhang L, Liu Z, Wang X, Geng S, Li J et al (2018) Predicting medication nonadherence risk in a Chinese inflammatory rheumatic disease population: development and assessment of a new predictive nomogram. Patient Prefer Adher 12:1757-1765

18. Chuang YC, Chermansky C, Kashyap M, Tyagi P (2016) Investigational drugs for bladder pain syndrome (BPS)/interstitial cystitis (IC). Expert Opin Investig Drugs 25(5):521-529

19. Berry SH, Elliott MN, Suttorp M, Bogart LM, Stoto MA, Eggers $P$ et al (2011) Prevalence of symptoms of bladder pain syndrome/ interstitial cystitis among adult females in the United States. J Urol 186(2):540-544

20. Byrne DS, Sedor JF, Estojak J, Fitzpatrick KJ, Chiura AN, Mulholland SG (1999) The urinary glycoprotein GP51 as a clinical marker for interstitial cystitis. J Urol 161(6):1786-1790

21. Keay SK, Zhang CO, Shoenfelt J, Erickson DR, Whitmore K, Warren JW et al (2001) Sensitivity and specificity of antiproliferative factor, heparin-binding epidermal growth factor-like growth factor, and epidermal growth factor as urine markers for interstitial cystitis. Urology 57(6 Suppl 1):9-14

22. Jacobs BL, Smaldone MC, Tyagi V, Philips BJ, Jackman SV, Leng WW et al (2010) Increased nerve growth factor in neurogenic overactive bladder and interstitial cystitis patients. Can J Urol 17(1):4989-4994

23. Kuo HC (2014) Potential urine and serum biomarkers for patients with bladder pain syndrome/interstitial cystitis. Int $\mathrm{J}$ Urol 21(Suppl 1):34-41

24. Magalhaes TF, Baracat EC, Doumouchtsis SK, Haddad JM (2019) Biomarkers in the diagnosis and symptom assessment of patients with bladder pain syndrome: a systematic review. Int Urogynecol J 30(11):1785-1794

25. Akiyama Y, Luo Y, Hanno PM, Maeda D, Homma Y (2020) Interstitial cystitis/bladder pain syndrome: the evolving landscape, animal models and future perspectives. Int J Urol 27(6):491-503

26. Duh K, Funaro MG, DeGouveia W, Bahlani S, Pappas D, Najjar $S$ et al (2018) Crosstalk between the immune system and neural pathways in interstitial cystitis/bladder pain syndrome. Discov Med 25(139):243-250

27. Wang C, Li Q, Song X, Dong X (2019) Bayesian adaptive lasso for additive hazard regression with current status data. Stat Med 38(20):3703-3718

28. Louzada F, Shimizu TK, Suzuki AK (2020) The Spike-and-Slab Lasso regression modeling with compositional covariates: an application on Brazilian children malnutrition data. Stat Methods Med Res 29(5):1434-1446

29. Vickers AJ, Elkin EB (2006) Decision curve analysis: a novel method for evaluating prediction models. Med Decis Making 26(6):565-574

30. Ho-Le TP, Tran HTT, Center JR, Eisman JA, Nguyen HT, Nguyen TV (2021) Assessing the clinical utility of genetic profiling in fracture risk prediction: a decision curve analysis. Osteoporos Int 32(2):271-280

Publisher's Note Springer Nature remains neutral with regard to jurisdictional claims in published maps and institutional affiliations. 